\title{
Letter to the Editor about the article "A systematic review and meta-analysis of the effect of bisphosphonate drug holidays on bone mineral density and osteoporotic fracture risk"
}

\author{
Mohammad eghbal Heidari ${ }^{1}$ \\ Received: 19 January 2019 / Accepted: 14 February 2019 / Published online: 2 August 2019 \\ (C) International Osteoporosis Foundation and National Osteoporosis Foundation 2019
}

Dear Editor,

We read with interest the recent article by S. Nayak et al. [1] who concluded in a systematic review and meta-analysis study that considered the use of bisphosphonate discontinuation for bone mineral density and osteoporotic fracture risk in women who do not have low hip BMD or who have low hip BMD. This finding is important because it may provide important effectiveness of bisphosphonate in women with low hip BMD or without it so we have some concerns.

First, about the adaptation of systematic review guidelines and registrations; there have been systematic review and metaanalysis protocol registration established that help to maintain a level of homogeneity and quality across all meta-analyses and systematic reviews being conducted. PRISMA, Cochrane, JBI, and MOOSE are a few examples of such guidelines [2-4]. Therefore, the lack of any mention of the use of such instructions, even though the study itself follows the core principles of a systematic review and meta-analysis established in such statements/guidelines, raises the question of whether any such guideline was not used or just not mentioned. Second, the authors assessed the quality of the cohort studies by Newcastle-Ottawa quality assessment scale [5]. The result about inter-rater reliability of this tool is different and ambiguous [6]. There is a disagreement between researchers that may affect the results of publications [7, 8]. Third, only four studies are clinical trials, and the limitation of evidence document may make a bias about the effectiveness of bisphosphonate in apositive or negative way, then the ethnic bias should be considered carefully. One of my concerns is that most studies conducted in the USA and studies

Mohammad eghbal Heidari

mohammad.eghbal.heydari@gmail.com

1 Students' Scientific Research center, Faculty of Nursing Midwifery, Tehran University of Medical Sciences, Tehran, Iran done with similar researchers may be a barrier to generalization to population. We should consider biases in this part of article. Finally, the purpose of the systematic review is to perform interventions in society, but we should consider the cost of drug intervention especially in a developed country for implementation. We hope that the authors address the points presented and that the overall discussion of the presented points will only serve to benefit the research community at large.

\section{References}

1. Nayak S, Greenspan S (2019) A systematic review and meta-analysis of the effect of bisphosphonate drug holidays on bone mineral density and osteoporotic fracture risk. Osteoporos Int 30(4):705-720

2. David M, Alessandro L, Jennifer T, Altman Douglas G (2009) Preferred reporting items for systematic reviews and meta-analyses: the PRISMA statement. Ann Intern Med 151(4):264-269

3. Joanna Briggs Institute (2014) Joanna Briggs Institute reviewers' manual: 2014 edition. The Joanna Briggs Institute, Australia

4. Stroup Donna F, Berlin Jesse A, Morton Sally C, Ingram O, David WG, Drummond R et al (2000) Meta-analysis of observational studies in epidemiology: a proposal for reporting. JAMA 283(15):20082012

5. Stang A, Jonas S, Poole C (2018) Case study in major quotation errors: a critical commentary on the Newcastle-Ottawa scale. Eur J Epidemiol 33(11):1025-1031

6. Oremus M, Oremus C, Hall GB, McKinnon MC (2012) ECT, Team CSR. Inter-rater and test-retest reliability of quality assessments by novice student raters using the Jadad and Newcastle-Ottawa Scales. BMJ Open 2(4):e001368

7. Lo CK-L, Mertz D, Loeb M (2014) Newcastle-Ottawa Scale: comparing reviewers' to authors' assessments. BMC Med Res Methodol 14(1):45

8. Stang A (2010) Critical evaluation of the Newcastle-Ottawa scale for the assessment of the quality of nonrandomized studies in meta-analyses. Eur J Epidemiol 25(9):603-605

Publisher's note Springer Nature remains neutral with regard to jurisdictional claims in published maps and institutional affiliations. 